\section{La administración andaluza elabora una estrategia integrada y transversal para la gestión del paisaje}

El pasado día 12 de enero culminó el proceso de elaboración de la Estrategia del Paisaje de Andalucía (EPA) con la valoración positiva de la Comisión Interdepartamental de Valoración Territorial y Urbanística y su elevación al Consejo de Gobierno de la Junta de Andalucia. En su desarrollo han participado todas las consejerías y agencias de la comunidad autónoma andaluza con competencias en acciones con repercusión en el paisaje a través de un grupo de trabajo creado a tal efecto liderado por las consejerías de Obras Públicas y Vivienda, de Cultura y de Medio Ambiente.

Con esta iniciativa se avanza en el cumplimiento de las determinaciones de integración, coordinación y transversalidad de las políticas públicas en materia de paisaje acordadas en el Convenio Europeo del Paisaje (Consejo de Europa, 2000), en vigor en España desde el 1 de marzo de 2008.

El contenido del documento se estructura en seis apartados principales. Los dos primeros aportan la justificación y oportunidad histórica de la Estrategia, así como el contexto normativo básico a nivel europeo y autonómico con relación o incidencia sobre los paisajes. El tercer capitulo detalla los principios rectores sobre los que descansa la Estrategia: a) gobernanza territorial, como guía de co-responsabilidad y co-participación pública y privada en la creación y aplicación de políticas en el territorio; b) desarrollo sostenible, como compromiso entre la sociedad y sus aspiraciones de desarrollo y el respeto a su entorno medioambiental; c) subsidiariedad, como vía de toma de decisiones y acciones en los niveles político-administrativos más próximos al ciudadano; d) y, por último, el principio combinado de la prevención y la precaución, en el sentido de priorizar más la actuación sobre las causas que sobre los efectos ya producidos en el paisaje, y en la contención de acciones ante la incertidumbre posible de unos resultados negativos para el paisaje.

El cuarto apartado se dedica a una exposición sintética del estado actual de los pai- sajes andaluces apuntando los procesos y tendencias que operan en la actualidad.

Los dos últimos integran los aspectos más operativos de la Estrategia. El capítulo quinto se centra en la definición de siete objetivos, la articulación de líneas estratégicas y la imbricación de una serie de medidas ordenadas en tres aspectos: la integración institucional/normativa, la planificación y gestión, y la actuación o ejecución. Este capitulo aporta unos de los más importantes valores de la Estrategia como es el compromiso institucional alcanzado estando presentes todos los ámbitos competenciales al nivel de la administración de la Junta de Andalucía. El sexto y último capítulo está dedicado a la elaboración, ejecución, seguimiento, evaluación y comunicación de la Estrategia como pilares de su puesta en práctica, así como el establecimiento del asesoramiento y acompañamiento científico.

Desde el punto de vista del desarrollo de contenidos directamente relacionados con las competencias exclusivas de la Consejería de Cultura, que ha contado con representación del IAPH y la Dirección General de Bienes Culturales, la Estrategia incluye un objetivo específico orientado a "impulsar la recuperación y mejora paisajística del patrimonio cultural", ordenado en dos líneas estratégicas:

> Valores culturales del paisaje: línea en la que tienen cabida aspectos de adecua- ción normativa, elaboración de directrices y recomendaciones sobre el análisis, diagnóstico y actuaciones sobre los paisajes en relación con los bienes protegidos y sus entornos. Supone, por otra parte, el impulso de las acciones de descontaminación visual y perceptiva, o la elaboración de guias de integración paisajística para dichos espacios e inmuebles, aspectos en los que tradicionalmente viene trabajando la Dirección General de Bienes Culturales, por ejemplo mediante los programas sobre protección de conjuntos históricos.

$>$ Paisajes de interés cultural: linea que supone, en primer lugar, un respaldo decidido a la metodología de registro y de definición de criterios para el estudio de los paisajes culturales de mayor calidad y singularidad patrimonial en el ámbito regional, labor que viene desarrollando por parte del IAPH. Esta línea estratégica implica la consideración de aspectos administrativos, como es la valoración de una posible figura de salvaguardia y fomento de los paisajes desde el campo del patrimonio histórico; también otros aspectos de gestión, como la creación de directrices y recomendaciones para la delimitación, gestión o integración de la participación social en paisajes de interés cultural; y, por último, aspectos de ejecución de programas tales como la elaboración del propio registro de paisajes de interés cultural, las guias de calidad paisajística de los paisajes de interés cultural, o el impulso de programas de difusión y comunicación de dichos paisajes.

Con este paso, Andalucia se sitúa en una posición adelantada en el panorama nacional, junto a otras comunidades, como Cataluña, Comunidad Valenciana, Islas Baleares, Galicia o Murcia, que cuentan con herramientas similares de vocación integradora y transversal destinadas a la aplicación del Convenio Europeo del Paisaje.

José María Rodrigo Cámara, Laboratorio del Paisaje, Centro de Documentación y Estudios del IAPH 